\title{
Automatic detection and recognition of silicosis in chest radiograph
}

\author{
Lei Zhu, Ran Zheng, Hai Jin *, Qin Zhang and Wei Zhang \\ Services Computing Technology and System Lab, Cluster and Grid Computing Lab, School of Computer \\ Science and Technology, Huazhong University of Science and Technology, Wuhan, 430074, China
}

\begin{abstract}
Silicosis remains one of the most harmful occupational respiratory diseases. It threatens the workers exposed to dust environment. Chest radiograph is the main available image source for silicosis diagnosis according to the diagnostic criteria of pneumoconiosis (DCP). Automatic detection and recognition of silicosis in chest radiograph has great importance on aiding the process of silicosis diagnosis. This paper proposes a multi-scale opacity detection approach to detect all suspected opacities from the chest radiograph. A support vector machine (SVM) based computer-aided silicosis diagnosis is proposed to recognize silicosis opacity from a large amount of candidate regions, and gives processing result for radiologist reference. Comprehensive experiments conducted on real world chest radiographs demonstrate that the proposed approach can reveal changes of silicosis pathology well, and it can be adopted as an effective tool for automatic silicosis diagnosis.
\end{abstract}

Keywords: Silicosis, diagnostic criteria of pneumoconiosis, chest radiograph, support vector machine, silicosis pathology

\section{Introduction}

Silicosis is still one of the most harmful occupational respiratory diseases in the world. A correct silicosis diagnosis has great meaning on timely medical treatment [1]. Diagnostic criteria of pneumoconiosis (DCP) indicates that posterior-anterior chest radiograph is the main available image source for silicosis diagnosis.

Chest check is one of the most common types of physical examination, since it is cost-effective, routinely available, and dose-effective. However, it is extremely challenging to interpret the recorded chest radiographs, since they contain complex visual contents of superimposed anatomical structures [2]. Even an experienced radiologist may have troubles on distinguishing infiltrates from normal patterns of branching blood vessel or detecting subtle opacity in the lung fields [3]. Silicosis opacities are usually quite small and thick, and they are distributed over lung field. These characteristics make the process of opacity detection and recognition in silicosis diagnosis time-consuming. Therefore, it has great importance to develop an automatic approach for accurate and fast opacity detection and recognition to aid and speedup the process of silicosis diagnosis.

\footnotetext{
*Corresponding author: Hai Jin, Services Computing Technology and System Lab, Cluster and Grid Computing Lab, School of Computer Science and Technology, Huazhong University of Science and Technology, Wuhan 430074, China. Tel.: +86-2787543529 ext 8033; Fax: +86-27-87557354; E-mail: hjin@ hust.edu.cn.
}

0959-2989/14/\$27.50 @ 2014 - IOS Press and the authors. 
However, for its low quality, raw medical image cannot be applied directly. It is a consensus that medical image processing has great importance on medical diagnosis. This paper proposes a multi-scale opacity detection approach to detect all suspected opacities from the chest radiograph, and support vector machine (SVM) [4] based model to recognize the correct one from the candidate ones. The proposed approach includes three main stages: opacity detection, opacity feature extraction, and opacity classification. Opacity detection is to detect all the candidate objects for further classification. Several visual features are extracted at the stage of opacity feature extraction to describe the visual contents of images. With the extracted features and SVM classifier, a discriminative classifier is learned for online classification at the stage of opacity classification.

The rest of this paper is structured as follows. The details of the proposed approach are introduced in Section 2. Experiments and results are presented in Section 3. Finally, conclusions are given in Section 4.

\section{The proposed approach}

\subsection{Opacity detection}

Due to the low quality of the raw image, lung region of interest (ROI) preprocessing is exploited in this paper to enhance the region of salient opacities at all scale of interest (SOI). This preprocessing has great impact on the precision of opacity detection. More specifically, this process can be further divided into three sub-processes: polynomial image fitting, histogram equalization, and multi-scale enhancement. Polynomial image fitting is utilized to remove lung structures with high gray level but low contrast. Histogram equalization is leveraged to enhance the contrast of local or global regions. Multi-scale enhancement is exploited to enhance the region of salient opacities of various sizes and different contrasts. Figure 1 presents the results obtained at all steps of image preprocessing.

Multi-scale enhancement is to produce an enhanced image via background subtraction, which is implemented by subtracting a smoothed version of the image from the original one, so that the high frequencies are highlighted. The result largely depends on the size of the operator used to produce the smoothed image. In this case, only a limited range of frequency is highlighted. A smoothing operator of a certain size is used to enhance the visibility of the opacities. Candidate objects are then detected by thresholding the enhanced images and measuring the shape.

For multi-scale enhancement, several Gaussian kernels should be first constructed at multiple scales. This procedure can be further divided into three sub-procedures: (1) Calculate the size of detection objects $d$ by counting the number of included pixels, and set the Gaussian kernel size as $d$. It is worthwhile to mention that size $d$ should be ignored if it is an even number, because the dimension of Gaussian kernel is an odd number. If an even $d$ is taken into count, Gaussian kernel dimension is $d+1$, which will cause repeated computations. (2) Generally, for a normal distribution $X \sim N\left(\mu, \sigma^{2}\right)$, the range $[-3 \mu, 3 \mu]$ covers almost all of effective filter coefficients. So in this paper, Gaussian filter dimension is set as $6 \sigma$. For dynamic Gaussian filter, standard deviation $\sigma$ and mean $\mu$ in kernel are set as $(d-1) / 6$, $d / 2$, respectively. (3) Finally, Gaussian filter function at scale $d$ is built as Eq. (1).

$$
G_{d}(x, y)=\frac{18}{\pi(d-1)^{2}} e^{\frac{9\left[(2 x-d)^{2}+(2 y-d)^{2}\right]}{2(d-1)^{2}}}
$$



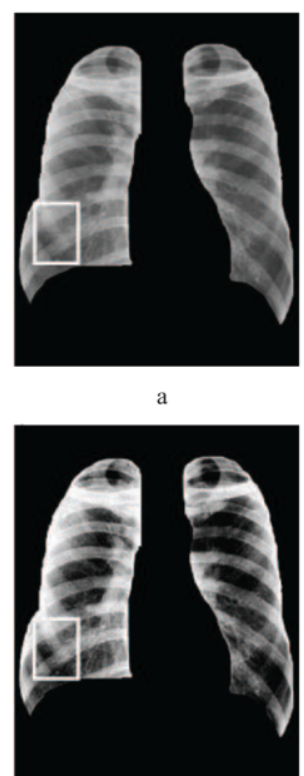

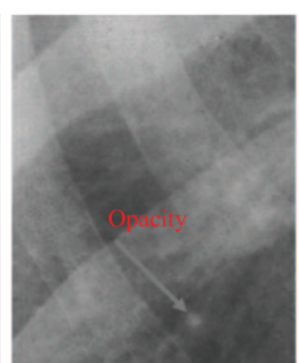

b

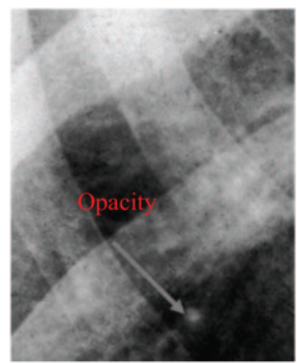

f

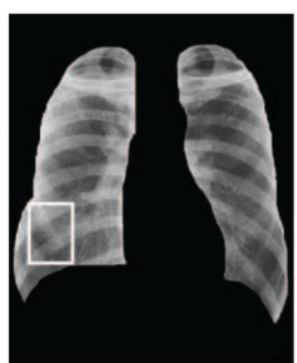

c

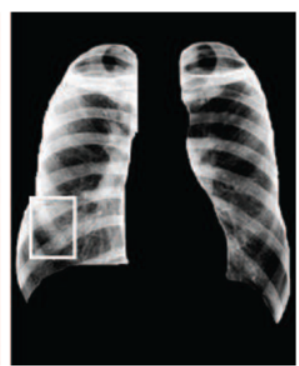

g

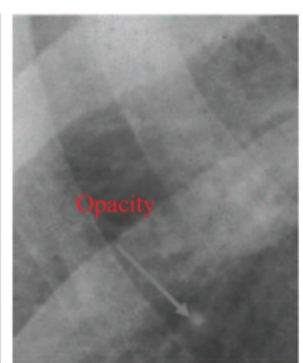

$\mathrm{d}$

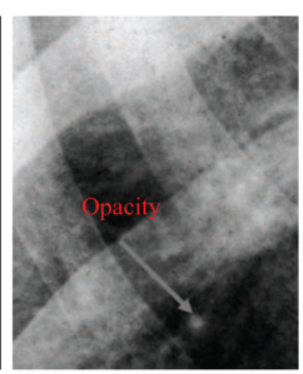

h

Fig. 1. Results of preprocessing. $\mathrm{a}$ is the original image. $\mathrm{c}$ describes the results of polynomial fitting. e describes the results of histogram equalization. $g$ describes the results of multi-scale enhancement. $b, d, f, h$ describe the enlarged view of the rectangle area in images a, c, e, g, respectively. Arrows in images b, d, f, h indicate the position of detected opacities.

After constructing the Gaussian kernels at multiple scales, the subtracted image is calculated by substraction technique. Traditional background subtraction techniques are based on temporal images. An input image is subtracted by its previous or next image to get the difference for object detection. In this paper, salient information such as pathological changes and opacities are needed. The smoothed version of original image is adopted as background, and the original image as foreground. The subtracted image is then calculated by subtracting background image from the foreground image. Via this procedure, high frequency information is reserved to the original image and the interested domain is greatly enhanced. The resulted image at scale $d$ is described as Eq. (2).

$$
D_{d}(x, y)=f(x, y)-f(x, y) * G_{d}(x, y)
$$

where $G_{d}(x, y)$ is the Gaussian kernel at scale $d$.

Assuming that the range of SOI is $\left[D_{\min }, D_{\max }\right]$, multi-scale enhancement scheme on chest radiograph follows the steps that are described as follows: (1) Produce Gaussian smooth kernel at scale $d(d \in$ [Dmin, Dmax]). (2) Obtain the subtracted image $D_{d}(x, y)$ by subtraction technique. (3) Exploit single threshold value to threshold $D_{d}(x, y)$ to generate a binary image $R_{d}(x, y)$. (4) Repeat the three above steps to get high frequency binary images at all even scale $d$. (5) Use OR operation to obtain a sum image of all binary images, and AND operation to implement the sum between the obtained sum image and original image. Its equation is shown in Eq. (3).

$$
R(x, y)=f(x, y) \quad \& \sum_{d=D_{\min }}^{D_{\max }} R_{d}(x, y)
$$




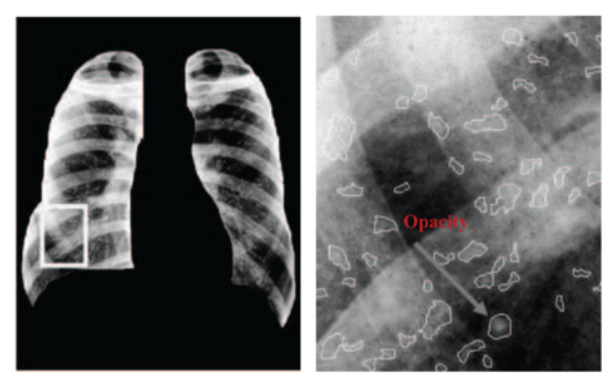

Fig. 2. Candidate opacity detection result. Right image is enlarged view of the rectangle area in left image (right line is 1:1.6 views of the actual image). Arrow indicates an opacity.

where $f(x, y)$ is original image, $\&$ is AND operation, $\sum$ is OR operation. According to the DCP, three SOI ranges of small opacity are $<1.5 \mathrm{~mm}, 1.5 \mathrm{~mm} \sim 3 \mathrm{~mm}$, and $3 \mathrm{~mm} \sim 10 \mathrm{~mm}$ in diameter. SOI of big opacity is above $10 \mathrm{~mm}$. If the size of big opacity is no smaller than $10 \mathrm{~mm} \times 20 \mathrm{~mm}$, a diagnostic conclusion of stage III silicosis can be drawn. Based on these ranges, SOI specific range can be obtained by mapping pixel space through points per inch (PPI) or pixel size of the input image. Since chest radiology cannot show lung tissues or abnormity that is no more than $1 \mathrm{~mm}$ in diameter, the final SOI is fixed as $1 \mathrm{~mm} \sim 20 \mathrm{~mm}$ in diameter, which constructs the basis of the range $\left[D_{\min }, D_{\max }\right]$. Figure 2 presents a typical opacity detection results.

\subsection{Opacity feature extraction}

In this paper, several features are extracted to describe the visual contents of the detected candidate opacities. Based on the extracted visual features, classifier model is learned to separate the positive and negative samples by a large margin. More specifically, these features include: normalized area, normalized perimeter, form ratio, circularity, areality, rectanglity, eccentricity, elongation. In addition to the eight basic visual features presented above, other features, lung bound overlap function ratio (LBF), lung bound overlap geometry ratio (LBG), and lung bound overlap circumcircle (LBC), are extracted to describe the location and scale features of opacities. Another nine features are calculated to describe gray level distribution of candidate opacity pixels. They are the mean, maximum, and minimum of gray value. Moreover, four contrast values are calculated between maximum gray value and mean, mean and minimum gray value, most pixel gray value and second most pixel gray, mean and two pixel thick irregular ring neighbor area of opacity. These features are all normalized by the maximum gray value.

\subsection{Opacity classification}

Large amount of candidate opacities leads to large false positives, which motivates us to exploit machine learning based approaches to classify the detected candidate opacities. In fact, almost all existing approaches for opacity detection have the problem of false positives. Machine learning based approach is one of the most effective approaches for false positives reduction with the least true positives mission. Almost all opacity detection approaches are based on two consecutive steps, which reduce the large false positives and extract the final opacity subset.

Over the past decades, SVM has been widely investigated and exploited in many different application fields and various classification tasks for its good performances $[5,6]$. In the field of medical image processing, several approaches have been proposed to employ SVM to achieve promising results. In this 


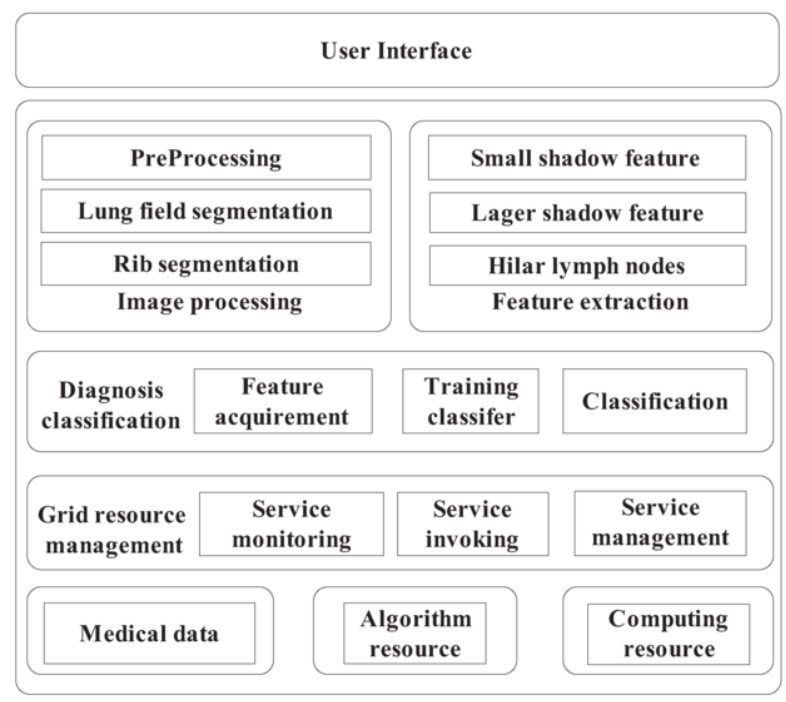

Fig. 3. Basic framework of the computer aided silicosis medical diagnosis system SisMed.

paper, SVM is used to learn the optimal classier on visual features extracted on the candidate set of opacities.

\section{Experiments and results}

The proposed approach is implemented in a computer aided silicosis medical diagnosis system, called as SisMed. Figure 3 shows its basic framework. In this system, the quality of raw image is first improved via image preprocessing. The candidate objects are then detected via the process of opacity detection. A SVM based recognition approach is proposed to recognize the possible objects. All the extracted features are concatenated to construct an unified feature vector, which is used to describe the visual contents of the objects. Some other tools for convenient use are also implemented. Each module of SisMed not only can be used as a single component, but also can be integrated together in any convenient way for automatic silicosis diagnosis. Therefore, SisMed could accomplish the whole silicosis recognition flow and present the diagnosis results to radiologist as opinion for reference. In this way, much screening work can be conducted in a batch way and the diagnosis process can be accelerated.

In this paper, experiments are conducted on three image datasets. Image set I contains 20 images from the standard pneumoconiosis radiograph set (only radiographs that can be used for silicosis diagnosis are included). Image set II contains images from the public JSRT database [7]. Image set III contains images from a private database, which includes 20 radiographs collected by Tongji Medical School of Huazhong University of Science and Technology (9 of them are annotated). Tables 1(a) and 1(b) present the details of image set I and image set II.

For image set I, accuracy of opacity classification is $85.7 \%$. For image set II, accuracy of big opacity classification is $83.3 \%$. For image set III, the accuracy of opacity classification is $77.8 \%$.

Accuracy of big opacity detection is tested on image set II, which is the standard digital image database for lung opacities and non-opacities. The first 12 images are applied in big opacity detection test. Table 2 shows the big opacity recognition test result. Details about opacities included in these images are not 
Table 1

Detailed descriptions for image sets used in experiments

(a) Detailed descriptions of the image set I

\begin{tabular}{ll}
\hline Description of image & Value \\
\hline Quantity & 20 \\
Width and height & $2435 \times 2337$ \\
Color & 256 gray level \\
Format &.$b m p$ \\
\hline
\end{tabular}

(b) Detailed descriptions of the image set II

\begin{tabular}{ll}
\hline Description of image & Value \\
\hline Quantity & 9 \\
Width and height & $2187 \times 2781$ \\
Color & 256 gray level \\
Format & .bmp \\
\hline
\end{tabular}

Table 2

Experimental results on big opacity classification

\begin{tabular}{lllll}
\hline Image filename & Number of opacity & $\begin{array}{l}\text { Number of recog- } \\
\text { nized big opacities }\end{array}$ & Correct or incorrect & Runtime (s) \\
\hline JPCLN001 & 1 & 8 & YES & 8 \\
JPCLN002 & 1 & 3 & YES & 6 \\
JPCLN003 & 1 & 2 & NO & 9 \\
JPCLN004 & 1 & 3 & YES & 4 \\
JPCLN005 & 1 & 1 & YES & 7 \\
JPCLN006 & 1 & 6 & NO & 7 \\
JPCLN007 & 1 & 7 & YES & 5 \\
JPCLN008 & 1 & 3 & YES & 4 \\
JPCLN009 & 1 & 2 & YES & 3 \\
JPCLN010 & 1 & 2 & YES & 6 \\
JPCLN011 & 1 & 5 & YES & 7 \\
JPCLN012 & 1 & 5 & YES & 6 \\
\hline
\end{tabular}

listed for protecting the privacy of patient. The big opacity recognition accuracy (named as BigAC) is calculated as Eq. (4).

$$
\text { BigAC }=\frac{\text { Number of images obtained correct big opacity }}{\text { Number of all images }}
$$

In this experiment, $B i g A C$ is $83.3 \%$ at the cost of $4.7 \mathrm{FP}$ (FP, false positives) per image on average. Average runtime of big opacity detection algorithm is six seconds.

Experiments are conducted on image set III to demonstrate the performance of the proposed approach on small opacity recognition. Table 3 shows the main experimental result. On seven images (out of nine images), the proposed approach achieves correct classification results. From the above presented results, a conjecture can be made that the proposed silicosis diagnosis approach can obtain desirable results.

\section{Conclusion}

This paper presents an effective approach to detect and recognize silicosis opacity. It completely obscures the rules of DCP, and is based on a common but effective recognition scheme. For preprocessing and candidate opacity recognition, multi-scale space produced by multi-scale filter is applied for various 
Table 3

Experimental results on image set III

\begin{tabular}{lll}
\hline Image ID & Standard result & Classification \\
\hline 1 & 1 & 1 \\
2 & 1 & 1 \\
3 & 2 & 2 \\
4 & 2 & 2 \\
5 & 0 & 1 \\
6 & 0 & 0 \\
7 & 0 & 1 \\
8 & 2 & 2 \\
9 & 2 & 2 \\
\hline
\end{tabular}

sizes of opacities. For feature extraction, a group of features aimed at various types of opacities is proposed and extracted for further classification. Based on the primary detection results, candidate opacities classification is applied for high FP reduction. The opacity detection and recognition technology is implemented in a practical computer-aided silicosis diagnosis system, SisMed. Comprehensive experiments on real medical images demonstrate the effectiveness of the proposed approach.

\section{Acknowledgment}

This work is supported by National Natural Science Foundation of China under grant No. 61133008 and the National High Technology Research and Development Program of China under grant 2012AA01A306.

\section{References}

[1] P.M. SaÌĄİA , A.C.D. Faria, A.S. Ferreira, A.J. Lopes, J.M. Jansen and P.L. Melo, Validation of the forced oscillation technique in the diagnostic of respiratory changes in patients with silicosis, Proceedings of IEEE International Conference on Engineering in Medicine and Biology Society (2010), 398-401.

[2] C.A. Mercan and M.S. Celebi, An approach for chest tube detection in chest radiographs, IET Image Processing 8 (2014), 122-129.

[3] S. Jaeger, A. Karargyris, S. Candemir, L. Folio, J. Siegelman, F. Callaghan, Z. Xue, K. Palaniappan, R.K. Singh, S. Antani, G. Thoma, Y.-X. Wang, P.-X. Lu and C.J. McDonald, Automatic tuberculosis screening using chest radiographs, IEEE Transactions on Medical Imaging 33 (2014), 233-245.

[4] C.-C. Chang and C.-J. Lin, LIBSVM: a library for support vector machines, ACM Transactions on Intelligent Systems and Technology 2 (2011), 27:1-27:27.

[5] F. Ye, Z. Zhang, K. Chakrabarty and X. Gu, Board-level functional fault diagnosis using multikernel support vector machines and incremental learning, IEEE Transactions on Computer-Aided Design of Integrated Circuits and Systems 33 (2014), 279-290.

[6] A. Bernieri, G. Betta, L. Ferrigno, M. Laracca and S. Mastrostefano, Multifrequency excitation and support vector machine regressor for ECT defect characterization, IEEE Transactions on Instrumentation and Measurement 63 (2014), 1272-1280.

[7] B. Van Ginneken, B.M. ter Haar Romeny and M.A. Viergever, Computer-aided diagnosis in chest radiography: a survey, IEEE Transactions on Medical Imaging 20 (2001), 1228-1241. 